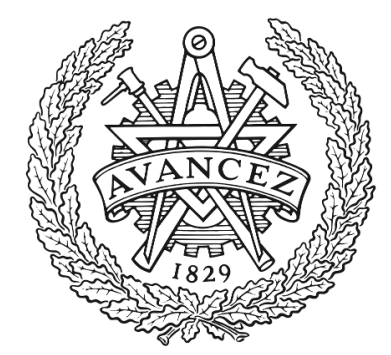

\title{
CHALMERS
}

UNIVERSITY OF TECHNOLOGY

\section{Analytical Solutions to Characteristic Impedance and Losses of Inverted Microstrip Gap Waveguide Based on Variational Method}

Downloaded from: https://research.chalmers.se, 2023-04-26 14:40 UTC

Citation for the original published paper (version of record):

Liu, J., Yang, J., Uz Zaman, A. (2018). Analytical Solutions to Characteristic Impedance and Losses of Inverted Microstrip Gap

Waveguide Based on Variational Method. IEEE Transactions on Antennas and Propagation, 66(12): 7049-7057. http://dx.doi.org/10.1109/TAP.2018.2869204

N.B. When citing this work, cite the original published paper.

(O2018 IEEE. Personal use of this material is permitted.

However, permission to reprint/republish this material for advertising or promotional purposes 


\title{
Analytical Solutions to Characteristic Impedance and Losses of Inverted Microstrip Gap Waveguide based on Variational Method
}

\author{
Jinlin Liu, Member, IEEE, Jian Yang, Senior Member, IEEE and Ashraf Uz Zaman, Member, IEEE,
}

\begin{abstract}
This paper initially introduces analytical solutions to newly invented inverted microstrip gap waveguide (IMGW). By applying the classical variational method the characteristic impedance, dielectric loss and conductor loss of the IMGW are obtained. The calculated characteristic impedance and losses of IMGWs with the present analytical method have been verified by commercial software CST. Furthermore, we have theoretically proved that the total loss of electromagnetic energy in the IMGW is much lower than that of covered microstrip line in millimeter waves (mmWs). Thereby, the IMGW has big advantages over covered microstrip lines for high-gain, high-efficiency array antennas in mmWs.
\end{abstract}

Index Terms-inverted microstrip gap waveguide, variational method, Green's function, characteristic impedance, dielectric loss and conductor loss.

\section{INTRODUCTION}

$\mathbf{M}$ OVING upwards in the spectrum for developing future wireless systems involves significant challenges from the hardware aspect [1]. Millimeter waves (mmWs) hardware modules need to be feasible for easy fabrication and high integration. Traditional hollow rectangular waveguide is normally manufactured in two parts and joined together. The two metallic structures usually suffer from the problem of poor electrical contacts. Another conventional transmission line, microstrip line, suffers from increased dielectric and radiation losses at high frequencies and spurious resonance when it is encapsulated or packaged. A covered microstrip line can overcome some of these disadvantages, but still presents high losses in the dielectrics in mmWs. Other solutions like substrate integrated waveguide (SIW) [2] exhibit the same unwanted losses in the dielectrics as frequency increases [3]. As a contrast, recently introduced gap waveguide technology [4]-[7] has low losses and low cost for manufacture. According to the theory of soft- and hard-surfaces [8], perfect electric conductor (PEC) and perfect magnetic conductor (PMC) parallel plates are able to create a frequency stopband where no waves can propagate through when the distance between the two plates is smaller than a quarter wavelength. As is well known, PMC does not exist in nature so that we apply metallic textured pins

This work is financially supported by the Swedish Governmental Agency for Innovation Systems VINNOVA via a project within the VINN Excellence center CHASE and the European Research Council (ERC) under 7th Framework Program ERC grant number 3222804.

The authors are with Department of Electrical Engineering, Chalmers University of Technology, Gothenburg, Sweden (e-mails: jinlin.liu@chalmers.se).

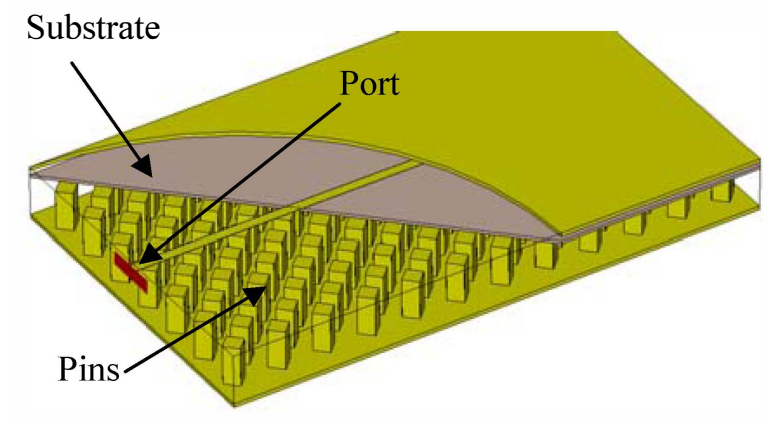

Fig. 1. 3-Dimensional geometry for IMGW.

surface for realizing an approximate PMC boundary condition.

There are four different realizations of gap waveguides - ridge gap waveguide, groove gap waveguide, inverted microstrip gap waveguide (IMGW) [6] and microstrip-ridge gap waveguide [9]. In the recent years some achievements on gap waveguide technology in the fields of passive element circuits [10]-[12], packaging technology [13] and high gain antennas [14]-[17] have been reported. Among the four types of the realized structures the IMGW has an advantage over the other three: its pin plate has a uniform pattern that can be easily manufactured. Fig. 1 illustrates a general IMGW based on the utilization of a thin substrate over a periodic pattern of metallic pins. This pin layer constitutes an approximate PMC surface [8], which together with the PEC plate creates a stopband. Nevertheless, a local quasi-TEM mode can propagate if there is a microstrip line within the structure. The big advantage of such a novel structure is that the metallic pin surface forces the electromagnetic wave propagates within the air gap between the microstrip and the top PEC plate so that high dielectric loss can be avoided in mmWs. Thereby, the IMGW has a much lower dielectric loss compared with traditional covered microstrip.

So far solving electromagnetic problems of the IMGW merely depends on the commercial electromagnetic (EM) solvers. Therefore, there are several uncertainties with only commercial numerical solvers so that an analytical solution is needed as a reference and base for the solutions of commercial solver. Firstly, until now, all numerical ports for gap waveguide are defined in such a way that the minimum reflection coefficient is achieved, where the value of the characteristic impedance is not emphasized. Therefore, we need an analytical method for the impedance as a reference to prove that our 
previous definition of the port for the IMGW is correct. Secondly, we would like to have formulas and figures for the dielectric loss and conductor loss of the IMGW in order to make a good design of high-gain antennas by using IMGW. The characteristic impedance and loss properties are key factors when an IMGW is utilized for high-gain antenna design in mmWs. Towards such kinds of themes of conventional microstrip lines there have been already an extensive amount of work done in the past decades - equivalent circuits model [18], theory of conformal mapping [19]-[21], perturbation method [22], variational method [23]-[25], spectral-domain method [26]-[28] and potential theory [29]-[30]. For a shielded microstrip line with an outer conductor it is convenient to establish a variational method and solve the corresponding Green's function in a closed form [25]. Nevertheless, it is not possible to obtain the Green's function in the closed form for the case of a parallel-plate transmission line with multilayered dielectrics. In [24] a variational method analysis for microstrip lines was carried out in spectral domain to determine the Green's function in a simple way. In this paper we apply the same variational method to the IMGW to calculate its impedance and losses. The same strategy is also applied to a conventional covered microstrip line so that comparison of the losses between the two types of transmission lines has been made directly in this work.

The organization of this paper is as follows. Firstly the Fourier transform is applied to both an ideal IMGW and a covered microstrip line, and the differential equation is solved by Green's function in spectral domain in Section II. Then, in Section III, theoretical analysis on characteristic impedance, dielectric loss and conductor loss are presented based on the analysis. Then, the corresponding simulated results by CST Microwave Studio are compared with the analytical solutions. Finally, conclusions and the limitations of this method are described.

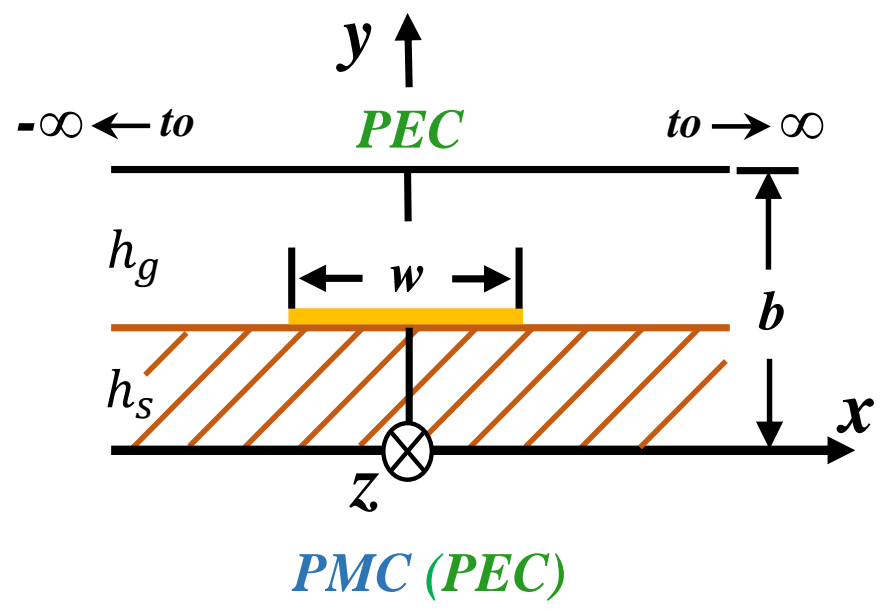

Fig. 2. The cross-sectional view of an ideal IMGW or a covered microstrip to be analyzed in this work. For a realistic IMGW case the metallic pin structure is treated as PMC boundary condition. For the covered microstrip line the bottom boundary condition is PEC.

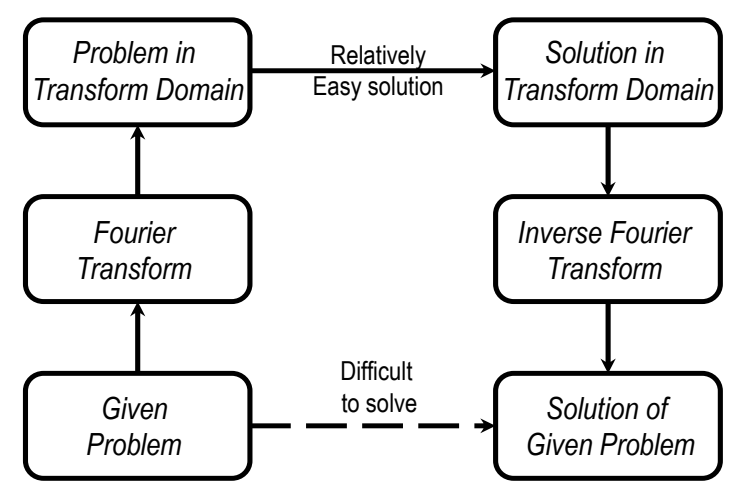

Fig. 3. Typical block diagram in Fourier transform method.

\section{FUndAMENTAL THEORY}

\section{A. Solutions by Spectral Domain Method}

According to [31], the TEM mode in a transmission line can be described by a model of electrostatic fields as the dominant mode, even when a nonuniform medium is applied in the structure. This kind of approximation has been proved to be reasonably accurate when the cross-sectional dimensions of a transmission line are smaller than the wavelength at the operation frequency. Therefore, the TEM mode problem can be treated as an electrostatic model, namely solving a Poisson's equation or a Laplace's equation. The cross section of an ideal IMGW to be analyzed in this paper is illustrated in Fig. 2. It is very difficult to directly solve Poisson's equation to the parallel plates IMGW where the boundary conditions along the $x$ axis are not specified (upper and bottom plates and the substrate are infinite along $x$ axis). Therefore, the utilization of Fourier transform in the second order differential equation will make its solution much simpler. The basic idea in the present method is illustrated in Fig. 3. Assuming the width of the strip $w<<\lambda$ and the height $b<\frac{\lambda}{4}$, where $\lambda$ is the wavelength at operation frequency so that electrostatic condition can be approximately fulfilled. The corresponding electric potential distribution $\psi(x, y)$ is related to the charge density $\rho(x, y)$ by the Poisson's equation,

$$
\nabla^{2} \psi(x, y)=\frac{\partial^{2} \psi(x, y)}{\partial x^{2}}+\frac{\partial^{2} \psi(x, y)}{\partial y^{2}}=-\frac{\rho(x, y)}{\varepsilon}
$$

where $\varepsilon$ is the permittivity of the substrate in the ideal IMGW. In reality, the thickness of microstrip is much smaller than the width $w$ so that the microstrip can be considered as infinitesimally thin and $\rho(x, y)$ can be described by

$$
\rho(x, y)=f(x) \delta\left(y-h_{s}\right)
$$

where $\delta\left(y-h_{s}\right)$ is the Dirac's function and $f(x)$ the charge density distribution on the microstrip.

The Green's function $G$, regarded as the potential due to a unit charge in an infinitely small volume at $\left(x^{\prime}, y^{\prime}\right)$, is the solution to the equation of

$$
\nabla^{2} G\left(x, x^{\prime} ; y, y^{\prime}\right)=-\frac{1}{\varepsilon} \delta\left(x-x^{\prime}\right) \delta\left(y-y^{\prime}\right)
$$


where $\delta\left(x-x^{\prime}\right) \delta\left(y-y^{\prime}\right)$ is the Dirac's function which expresses a unit charge. Once the Green's function $G$ is obtained, the electric potential $\psi(x, y)$ due to the charge distribution $\rho\left(x^{\prime}, y^{\prime}\right)$ can be determined by the superposition principle expressed as:

$$
\psi(x, y)=\int_{l^{\prime}} G\left(x, x^{\prime} ; y, y^{\prime}\right) \rho\left(x^{\prime}, y^{\prime}\right) d l^{\prime}
$$

where the integral is defined over the conductor contour $l^{\prime}$ in 2-D cross sectional structure. Now we apply the Fourier transform to convert the 2-D problem to a spatial 1-D problem in spectral domain,

$$
\widetilde{\psi}(k, y)=\int_{-\infty}^{\infty} \psi(x, y) e^{-j k x} d x .
$$

According to the differential property of the Fourier transform, namely, $\frac{\partial^{2} \psi(x, y)}{\partial x^{2}} \Leftrightarrow(j k)^{2} \widetilde{\psi}(k, y)$, Eq.(1) can be written in spectral domain as:

$$
-k^{2} \widetilde{\psi}(k, y)+\frac{\partial^{2} \widetilde{\psi}(k, y)}{\partial y^{2}}=0 \quad\left(y \neq h_{s}\right) .
$$

In spectral domain the solution of the potential distribution on the microstrip can be expressed as:

$$
\widetilde{\psi}(k, y)=\widetilde{f}(k) \widetilde{G}(k, y),
$$

where $\widetilde{f}(k)$ and $\widetilde{G}(k, y)$ are the charge density and the Green's function in spectral domain. Now we have already converted the second-order, source excited differential equation to a standard Sturm-Liouville problem [32], as expressed in (6). Then the corresponding boundary conditions will be applied to determine the Green's functions. The boundary conditions for the ideal IMGW structure (upper PEC and bottom PMC) illustrated in Fig. 2 are given as follows:

$$
\begin{array}{r}
\frac{\partial \widetilde{\psi}_{i}(k, 0)}{\partial y}=0 \\
\widetilde{\psi}_{i}(k, b)=0 \\
\varepsilon_{s} \frac{\partial \widetilde{\psi}_{i}\left(k, h_{s}^{-}\right)}{\partial y}-\varepsilon_{0} \frac{\partial \widetilde{\psi}_{i}\left(k, h_{s}^{+}\right)}{\partial y}=\widetilde{\rho}(k, y) \\
\widetilde{\psi}_{i}\left(k, h_{s}^{+}\right)=\widetilde{\psi}_{i}\left(k, h_{s}^{-}\right)
\end{array}
$$

where $\varepsilon_{s}$ stands for the permittivity of the substrate materials, $\widetilde{\psi}_{i}$ the electric potential in spectral domain of the IMGW. We utilize boundary conditions (8) to obtain the Green's function from (6), which leads to (10) and (11), where $\widetilde{G}_{i, s}(k, y)$ and $\widetilde{G}_{i, g}(k, y)$ are the Green's functions in spectral domain of the IMGW in the substrate and the air gap, respectively.

Similarly, the boundary conditions for the covered microstrip line structure (upper PEC and bottom PEC) illustrated in Fig. 2 are given as follows:

$$
\begin{array}{r}
\widetilde{\psi}_{c}(k, 0)=0 \\
\widetilde{\psi}_{c}(k, b)=0 \\
\varepsilon_{s} \frac{\partial \widetilde{\psi}_{c}\left(k, h_{s}^{-}\right)}{\partial y}-\varepsilon_{0} \frac{\partial \widetilde{\psi}_{c}\left(k, h_{s}^{+}\right)}{\partial y}=\widetilde{\rho}(k, y) \\
\widetilde{\psi}_{c}\left(k, h_{s}^{+}\right)=\widetilde{\psi}_{c}\left(k, h_{s}^{-}\right)
\end{array}
$$

where $\widetilde{\psi}_{c}$ is the electric potential in spectral domain of the covered microstrip line. From (6) and (9), we obtain the Green's functions for the covered microstrip line presented in (12) and (13), where $\widetilde{G}_{c, s}(k, y)$ and $\widetilde{G}_{c, g}(k, y)$ stand for the Green's functions in spectral domain of the covered microstrip line in the substrate and the air gap, respectively. Then, the electric potential in (6) can be expressed by inverse Fourier transform as:

$$
\psi(x, y)=\frac{1}{2 \pi} \int_{-\infty}^{\infty} \tilde{f}(k) \widetilde{G}(k, y) e^{j k x} d k
$$

\section{B. Characteristic Impedance of the IMGW}

The characteristic impedance is the ratio of the amplitudes of the voltage and the current on the transmission line. In the TEM mode approximation the characteristic impedance $Z_{0}$ [25] is usually evaluated by the line capacitance per unit length $C$

$$
Z_{0}=\frac{1}{c \sqrt{C_{0} C}}
$$

where $c$ is the velocity of light in free space and $C_{0}$ is the line capacitance per unit length for the case $\varepsilon_{s}=\varepsilon_{0}$. Here, the corresponding line capacitance per unit length $C$ is given by [31],

$$
C=\frac{Q^{2}}{\int_{-\infty}^{\infty} f(x) \psi\left(x, h_{s}\right) d x} \quad[F / m]
$$

We have already obtained the Green's functions in spectral domain. Then it is more convenient to evaluate the line

$$
\begin{array}{ll}
\widetilde{G}_{i, s}(k, y)=\frac{\sinh \left(k h_{g}\right) \cosh (k y)}{k\left[\varepsilon_{0} \cosh \left(k h_{s}\right) \cosh \left(k h_{g}\right)+\varepsilon_{s} \sinh \left(k h_{s}\right) \sinh \left(k h_{g}\right)\right]} & 0 \leq y \leq h_{s} \\
\widetilde{G}_{i, g}(k, y)=\frac{\cosh \left(k h_{s}\right) \sinh (k(b-y))}{k\left[\varepsilon_{0} \cosh \left(k h_{s}\right) \cosh \left(k h_{g}\right)+\varepsilon_{s} \sinh \left(k h_{s}\right) \sinh \left(k h_{g}\right)\right]} & h_{s} \leq y \leq b \\
\widetilde{G}_{c, s}(k, y)=\frac{\sinh \left(k h_{g}\right) \sinh (k y)}{k\left[\varepsilon_{0} \sinh \left(k h_{s}\right) \cosh \left(k h_{g}\right)+\varepsilon_{s} \cosh \left(k h_{s}\right) \sinh \left(k h_{g}\right)\right]} & 0 \leq y \leq h_{s} \\
\widetilde{G}_{c, g}(k, y)=\frac{\sinh \left(k h_{s}\right) \sinh (k(b-y))}{k\left[\varepsilon_{0} \sinh \left(k h_{s}\right) \cosh \left(k h_{g}\right)+\varepsilon_{s} \cosh \left(k h_{s}\right) \sinh \left(k h_{g}\right)\right]} & h_{s} \leq y \leq b
\end{array}
$$


capacitance per unit length in spectral domain, which can be obtained by utilizing the Parseval's theorem,

$$
C=\frac{2 \pi Q^{2}}{\int_{-\infty}^{\infty} \widetilde{f}(k) \widetilde{\psi}\left(k, h_{s}\right) d k} \quad[F / m]
$$

where $\widetilde{\psi}\left(k, h_{s}\right)=\widetilde{f}(k) \widetilde{G}\left(k, h_{s}\right)$ and $\widetilde{G}\left(k, h_{s}\right)$ is the Green's function evaluated at $y=h_{s}$,

$$
\begin{aligned}
& \widetilde{G}\left(k, h_{s}\right)= \\
& \frac{\sinh \left(k h_{g}\right) \cosh \left(k h_{s}\right)}{k\left[\varepsilon_{0} \cosh \left(k h_{s}\right) \cosh \left(k h_{g}\right)+\varepsilon_{s} \sinh \left(k h_{s}\right) \sinh \left(k h_{g}\right)\right]} .
\end{aligned}
$$

And the total charge per unit length $Q$ on the microstrip is given by,

$$
Q=\int_{-w / 2}^{w / 2} f(x) d x . \quad[C / m]
$$

\section{Discussion of Charge Density}

So far the charge density $f(x)$ has not been considered because it is still unknown. According to Thomson's theorem, the charges distribute themselves on the conducting structures so that the electric energy is minimized. In order to accurately calculate the line capacitance, two trial functions of the charge density distributions on the microstrip are discussed in this work. In the case of wide microstrip [25], the electrons are usually concentrated on the corners at both sides because of Coulomb's force among the electrons. Therefore, the electron density varies rapidly along the microstrip to its edge. However, the electron density distribution is more uniform when the width of the microstrip is small [24]. Accordingly, two normalized approximate functions $f_{\text {wide }}(x)$ and $f_{\text {narrow }}(x)$ of the charge density are chosen as follows,

$$
\begin{aligned}
& f_{\text {wide }}(x)=0.5+\left|\frac{x}{w}\right|^{3}, \\
& \qquad|x| \leq \frac{w}{2} \quad \text { and } \quad 0.3 \mathrm{~mm} \leq w \leq 3 \mathrm{~mm} \\
& f_{\text {narrow }}(x)=\left|\frac{x}{w}\right|, \quad|x| \leq \frac{w}{2} \quad \text { and } \quad w \leq 0.3 \mathrm{~mm}
\end{aligned}
$$

The corresponding Fourier transform expressions $\widetilde{f}_{\text {wide }}(k)$ and $\widetilde{f}_{\text {narrow }}(k)$ are as follows:

$$
\begin{aligned}
& \widetilde{f}_{\text {wide }}(k)=\frac{1}{k} \sin (k w / 2) \\
& +\frac{2}{k^{2} w}\left[\cos (k w / 2)-\frac{2 \sin (k w / 2)}{k w / 2}+\frac{\sin ^{2}(k w / 4)}{(k w / 4)^{2}}\right] \\
& \tilde{f}_{\text {narrow }}(k)=\frac{\sin (k w / 2)}{k w / 2}-\frac{\sin ^{2}(k w / 4)}{(k w / 4)^{2}}
\end{aligned}
$$

\section{Attenuation}

Theoretically, the loss components of a transmission line include dielectric loss and conductor loss. The attenuation constant of a transmission line due to the conductor loss can be obtained by the following [25]:

$$
\alpha_{c}=\frac{R_{s} \int_{l} i_{s}^{2} d l}{2 \int_{S} v \varepsilon(\nabla \psi)^{2} d S} \quad[\text { Neper/Unit Length }]
$$

where $R_{s}=\sqrt{0.5 \omega \mu_{0} / \sigma_{c}}$ is the surface resistance, $\sigma_{c}$ the conductivity of the microstrip, $i_{s}=v \rho\left(x, h_{s}\right)$ the current density on the microstrip, and $v=c \sqrt{C_{0} / C}$ the propagation velocity. The integral in the numerator is defined the contour $l$ of the microstrip and the integral in the denominator is defined over the whole cross-sectional area $S$. Notice that the unit of the conductor loss here is Neper/Unit Length, where 1 Neper $=8.6859 \mathrm{~dB}$. Similarly, the attenuation constant due to the dielectric loss can be calculated by [25]:

$$
\alpha_{d}=\frac{\sigma_{d} \int_{S}(\nabla \psi)^{2} d S}{2 \int_{S} v \varepsilon(\nabla \psi)^{2} d S} \quad[\text { Neper } / \text { Unit Length }]
$$

where the integral is defined over the whole cross-sectional area $S$ and $\sigma_{d}$ the conductivity of the dielectric material. The dielectric loss depends on not only the permittivity and the conductivity of the substrate, but also the ratio of the area of the substrate to the area of the air.

\section{Theoretical And Simulated Results}

In this section several numerical models are set up in CST Microwave Studio with the port defined as in [33] so that the comparisons between the analytical solutions presented in the previous section and the CST simulations can be used as a verification of the correctness of both the analytical method
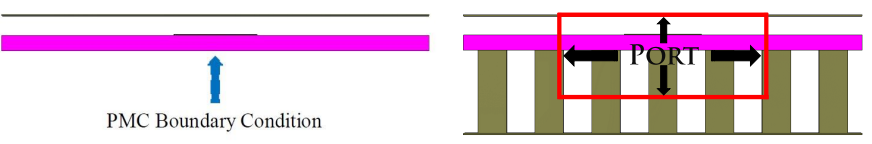

Fig. 4. The left figure illustrates a covered microstrip line with PMC boundary condition at the bottom. In the right one the PMC boundary condition is replaced by metallic pins.

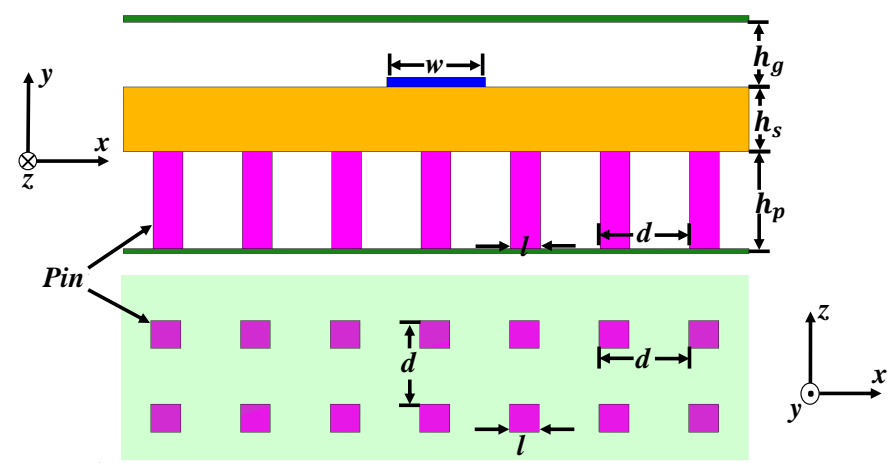

Fig. 5. The illustration of detailed geometrical dimensions utilized for the following research. 
TABLE I

GeOMetrical PARAmeters of THE STRUCTURE IN THE Fig.5 IN KA-,V- AND E-BANDS

\begin{tabular}{cccc}
\hline \hline Parameters[mm]/Frequency Bands & Ka-Band & V-Band & E-Band \\
\hline$d$ & 2.4 & 1.1 & 0.8 \\
\hline$l$ & 1.2 & 0.4 & 0.3 \\
\hline$h_{p}$ & 1.5 & 1.1 & 0.55 \\
\hline$h_{s}$ & 0.3 & 0.4 & 0.2 \\
\hline$h_{g} w$ & 0.4 & 0.25 & 0.15 \\
\hline$w$ & 1.2 & 1 & 0.8 \\
\hline \hline
\end{tabular}

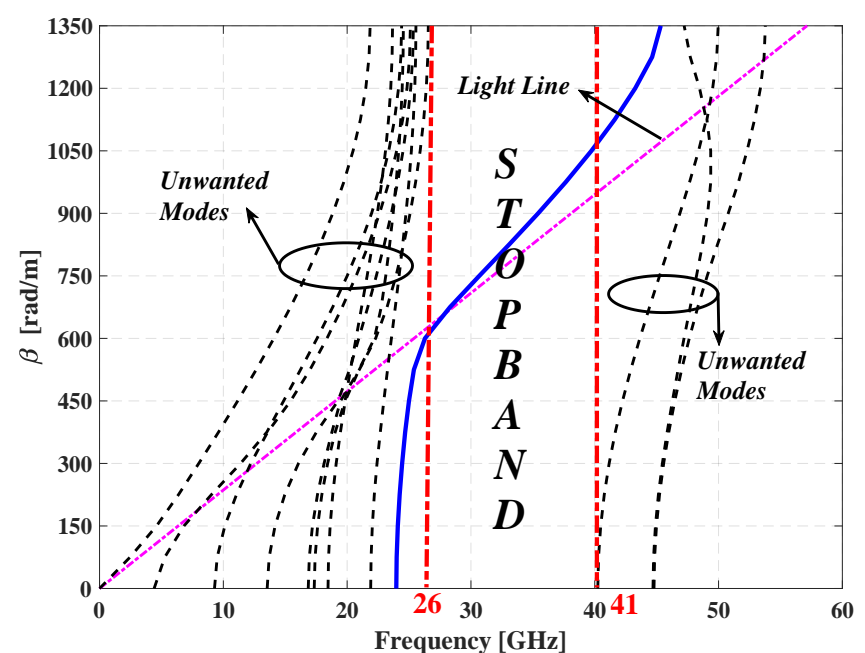

Fig. 6. The simulated dispersion diagram produced by CST for the IMGW of Ka-band model with geometrical dimensions defined in TABLE I. The solid blue curve indicates the quasi-TEM mode.

and numerical port definition if they are agreed with each other. As is shown in Fig. 4, we set up a simple straight covered microstrip line with a PMC boundary condition at the bottom. Then real metallic pins are modeled to realize the PMC boundary condition. The characteristic impedances of those two cases can be numerically evaluated from numerical ports in the CST Microwave Studio. An optimized waveguide port in [33] was applied for the IMGW case in order to minimize the reflection coefficient. We set up models in Kaband, V-band and E-band in this work. The 3-D geometrical dimensions for the corresponding structures are depicted in Fig. 5 and TABLE I. The substrate material utilized in simulations is Rogers RO4003C with relative permittivity $\varepsilon_{s}=$ 3.55 and loss tangent $\tan \delta=0.01$ at $60 \mathrm{GHz}$. RO4003C has advantages to be utilized in high-gain array antenna design [17] and microwave filter [10] because it is mechanically rigid enough and has a smaller loss value than traditional substrate FR4.

\section{A. Theoretical and simulated characteristic Impedances}

The dispersion diagram of the Ka-band structure produced by CST is illustrated in Fig. 6, where only the quasi-TEM mode propagates through the stopband from 26 to $41 \mathrm{GHz}$. Fig. 7 illustrates the comparison of characteristic impedances

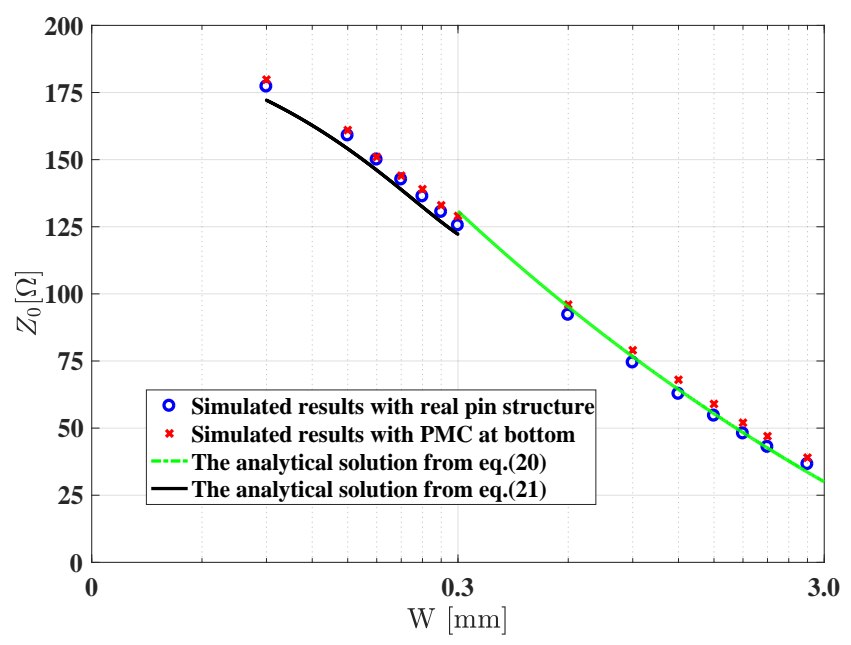

Fig. 7. Comparison of the present analytical solution with simulated results with real pin structure and those with PMC boundary condition at the bottom for Ka-band model with dimensions defined in TABLE I except for varied $w$.

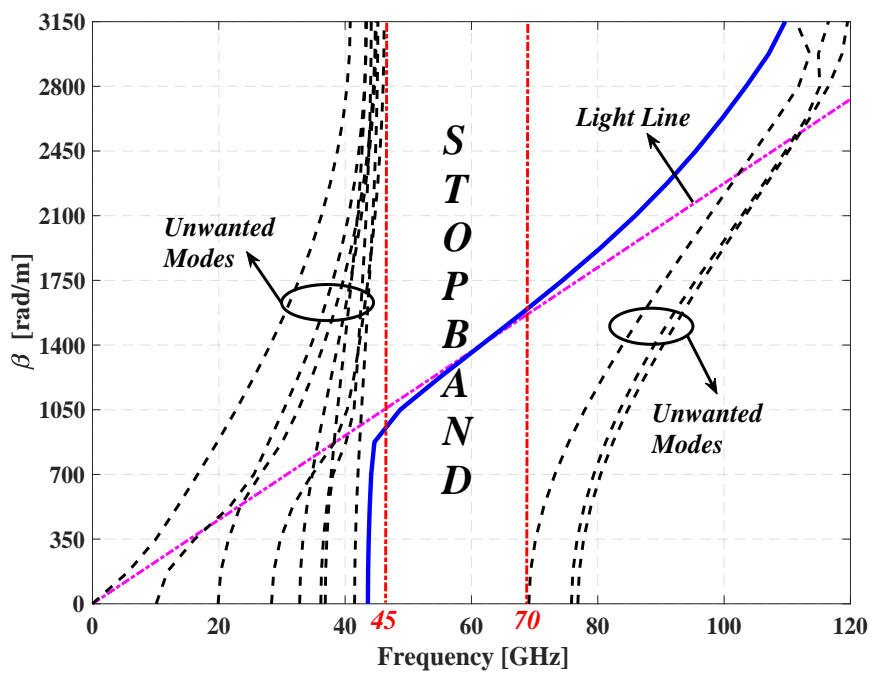

Fig. 8. The simulated dispersion diagram produced by CST for the IMGW of V-band model with geometrical dimensions defined in TABLE I. The solid blue curve indicates the quasi-TEM mode.

obtained between the present analytical solution, the simulated results of ideal IMGW and the IMGW realized with pin structure. The Ka-band model with dimensions is defined in TABLE I except for varied $w$. From the figure we can see that the analytical solution has good agreements with the 


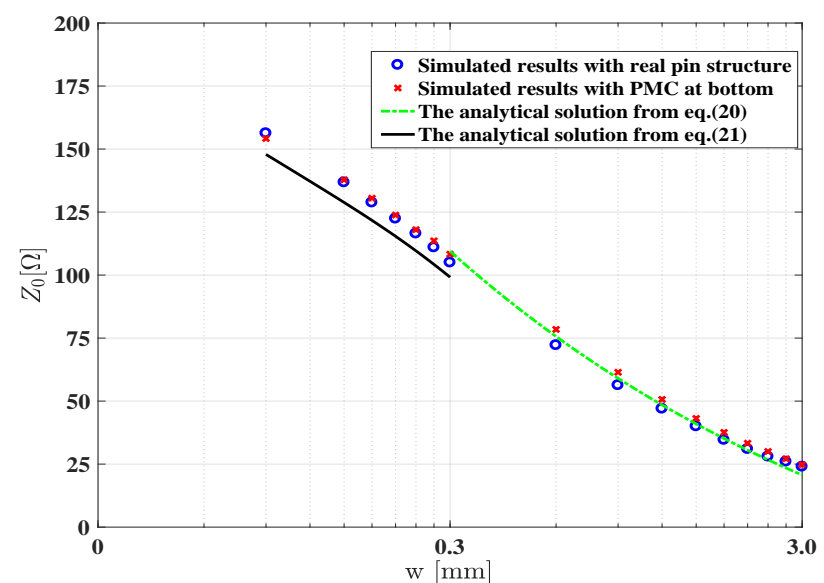

Fig. 9. Comparison of the present analytical solution with simulated results with real pin structure and those with PMC boundary condition at the bottom for V-band model with dimensions defined in TABLE I except for varied $w$.

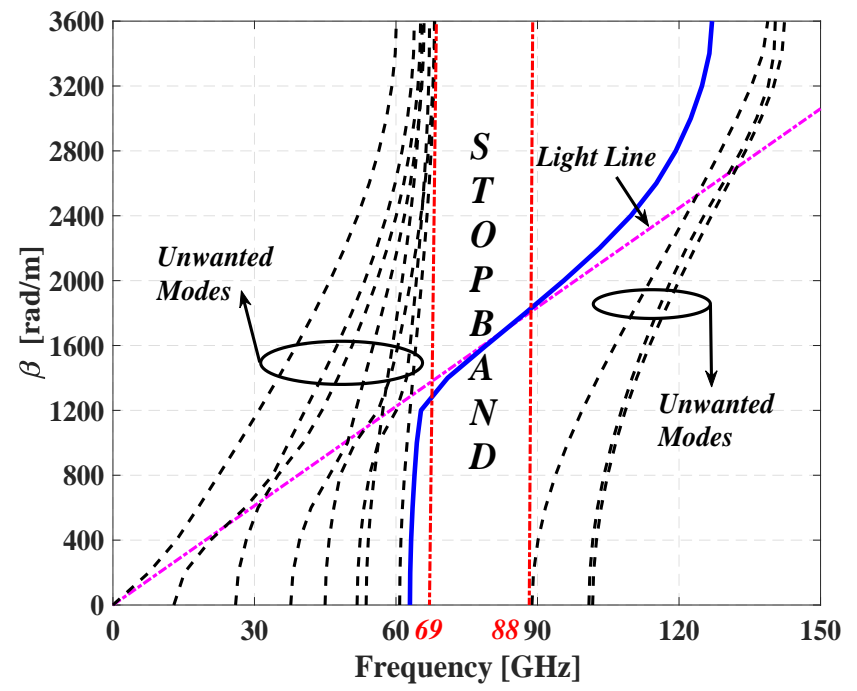

Fig. 10. The simulated dispersion diagram produced by CST for the IMGW of E-band model with geometrical dimensions defined in TABLE I. The solid blue curve indicates the quasi-TEM mode.

simulated results when the width of the IMGW $w$ is from $0.09 \mathrm{~mm}$ to $3 \mathrm{~mm}$. The relative error of the analytical solution of impedance is about 3\%-8\%. Similarly, Fig. 8 and Fig. 10 depict the dispersion diagrams of the models in both V-band and E-band, which are from 45 to $70 \mathrm{GHz}$ and 69 to $88 \mathrm{GHz}$. The corresponding analytical solutions of impedance also have good agreements with the simulated outcomes, as depicted in Fig. 9 and Fig. 11.

\section{B. Theoretical and simulated Attenuations}

The attenuation analysis is carried out from 54 to $70 \mathrm{GHz}$, for the $\mathrm{V}$ - band model as defined in Fig. 5 and TABLE I, with $w=1 \mathrm{~mm}$ and varied $h_{s}$ from $0.2 \mathrm{~mm}$ to $1 \mathrm{~mm}$. The theoretical dielectric losses of the covered microstrip line and

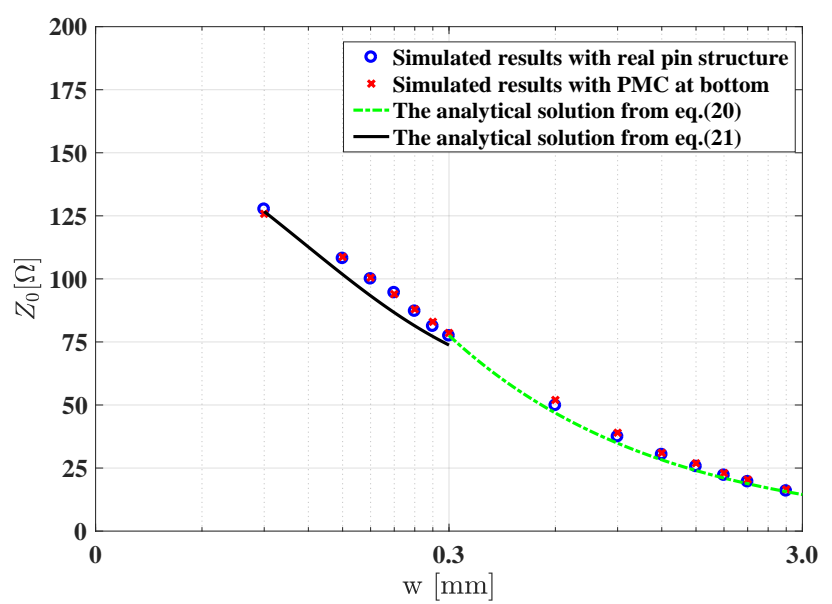

Fig. 11. Comparison of the present analytical solution with simulated results with real pin structure and those with PMC boundary condition at the bottom for E-band model with dimensions defined in TABLE I except for varied $w$.

the ideal IMGW are depicted in Fig. 12. In the figure, the attenuation constant of dielectric loss in the covered microstrip line increases with the thickness of the substrate, while that value of the IMGW is almost constant versus the thickness of the substrate. According to Fig. 12, the attenuation constant of dielectric loss in the covered microstrip line is around 10 times bigger than that value of the IMGW when the thickness of the substrate is $0.4 \mathrm{~mm}$. In order to determine the conductor losses of both structures, we assume that copper $\left(5.813 \times 10^{7}\right.$ $\mathrm{S} / \mathrm{m})$ microstrip, aluminum $\left(3.816 \times 10^{7} \mathrm{~S} / \mathrm{m}\right)$ metallic pins and plates are applied in this work. The corresponding conductor losses of the covered microstrip line and the IMGW according to (24) are evaluated and illustrated in Fig. 13. As is depicted, the conductor losses of the IMGW and the covered microstrip

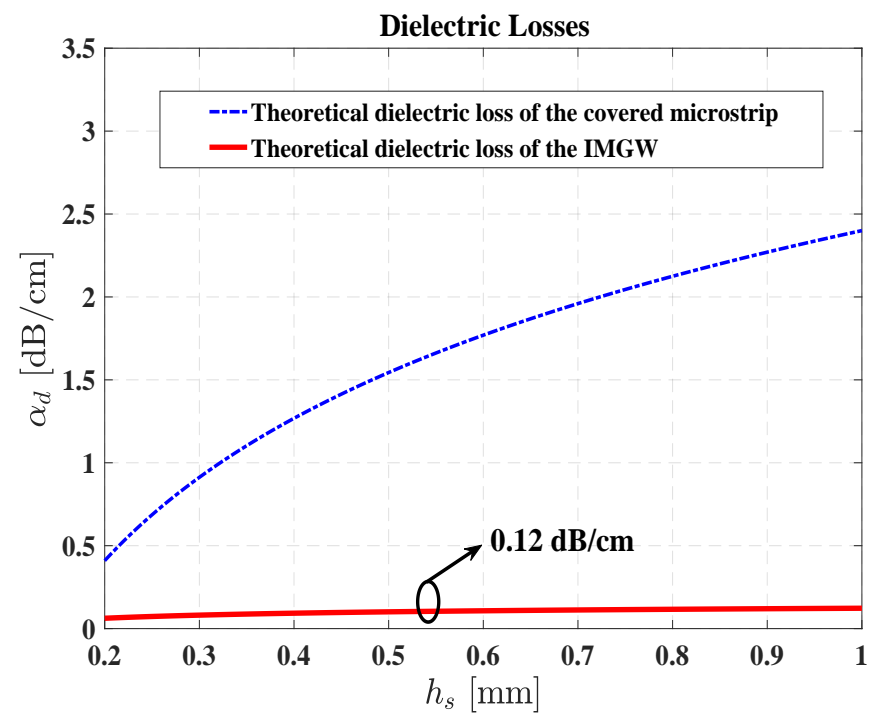

Fig. 12. Analytically calculated dielectric losses $\alpha_{d}$ of the V-band model of the covered microstrip line and the IMGW versus the substrate thickness $h_{s}$. The corresponding geometrical dimensions are defined in TABLE I. 


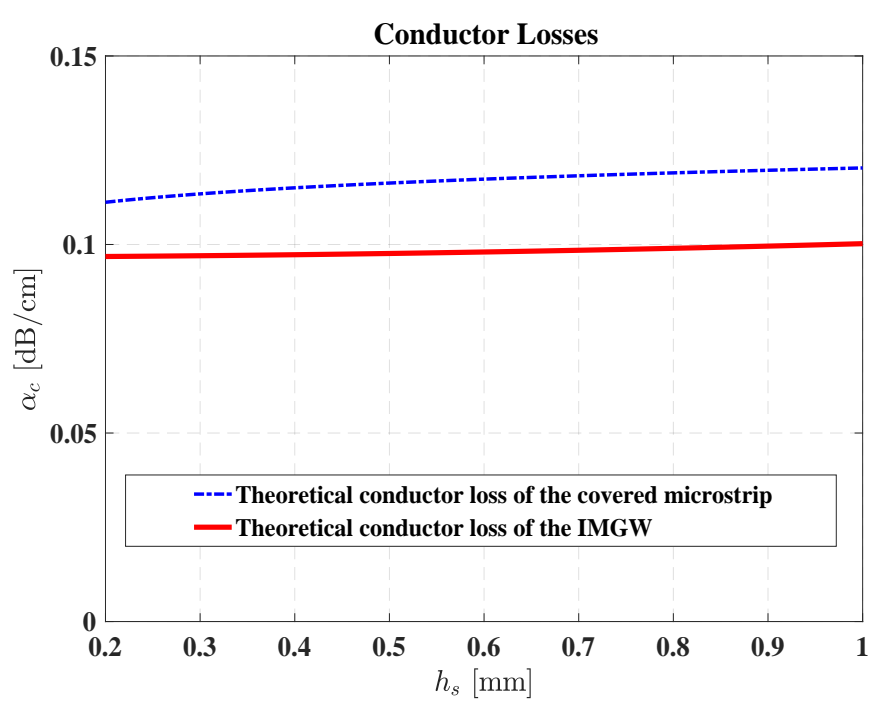

Fig. 13. Analytically calculated conductor losses $\alpha_{c}$ of the V-band model of the covered microstrip line and the IMGW versus the substrate thickness $h_{s}$. The corresponding geometrical dimensions are defined in TABLE I.

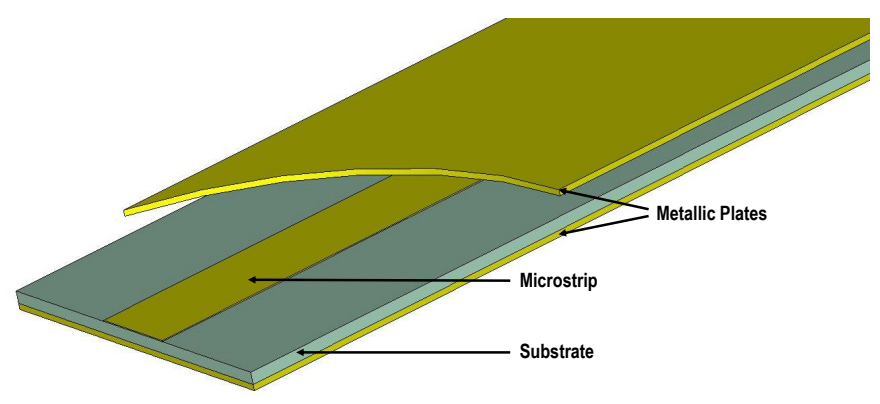

(a)

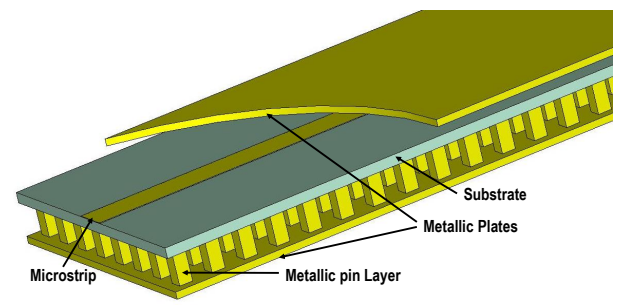

(b)

Fig. 14. The simulated structures of the covered microstrip line and the IMGW in order to compare their conductor losses and dielectric losses. Both of them have the same thickness of the substrate $h_{s}=0.4 \mathrm{~mm}$, length $L=$ $10 \mathrm{~mm}$, Width $W=9.9 \mathrm{~mm}$ and the width of the microstrip $w=1.0 \mathrm{~mm}$. (a) The covered microstrip line and (b) The IMGW.

line are both around $0.1 \mathrm{~dB} / \mathrm{cm}$. Since the surface resistances of both structure are identical and the charge densities inside the microstrips are assumed to be same in this work, the outcome depicted in Fig. 13 is also reasonable. Thus, the IMGW theoretically has large advantages than normal microstrip line or the covered microstrip line [34] in mmWs.

In order to verify our present analysis, a straight covered microstrip line and an IMGW with the same substrate and dimensions have been modeled in CST Microwave studio, as illustrated in Fig. 14. Transmission coefficients $S_{21}$ with lossless materials (PEC and lossless substrate) of two structures are firstly simulated. Then the structure with a PEC microstrip and lossy substrate are simulated again so that the difference of the two scenarios is the dielectric loss. As depicted in Fig. 15, the dielectric loss of the covered microstrip line is around $1.15 \mathrm{~dB} / \mathrm{cm}$ in V-band. The corresponding calculated value is $1.2 \mathrm{~dB} / \mathrm{cm}$ according to our theory in second section. Secondly, the dielectric loss of IMGW is illustrated in Fig. 16. The simulated value is about $0.22 \mathrm{~dB} / \mathrm{cm}$, and the calculated result is around $0.15 \mathrm{~dB} / \mathrm{cm}$. Note that the loss tangent value $\tan \delta$ is set up as 0.01 in CST Microwave Studio at $60 \mathrm{GHz}$ according to the Rogers [35]. The simulated value is a little bigger than the calculated one because the metallic pin surface

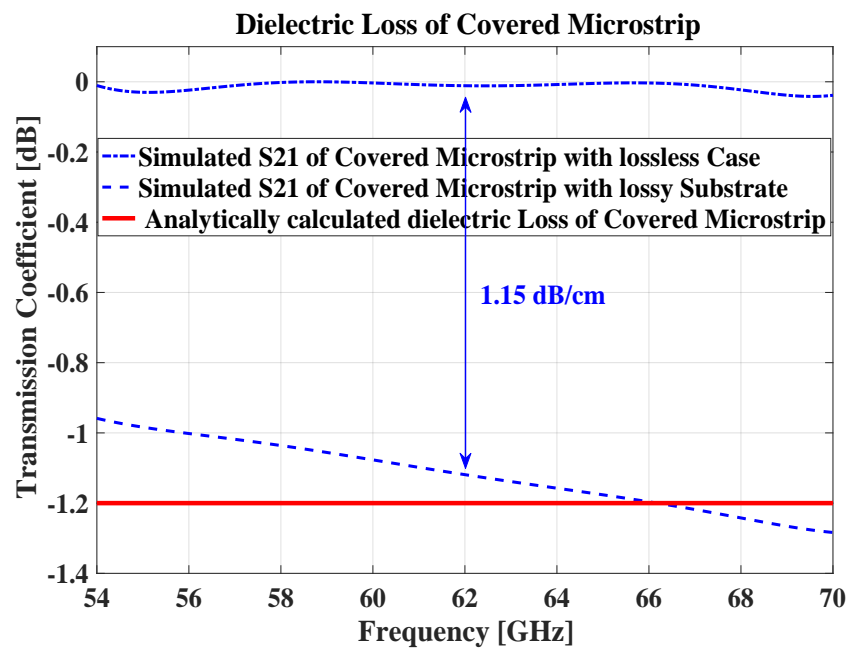

Fig. 15. The Simulated and the calculated dielectric losses of the covered microstrip line.

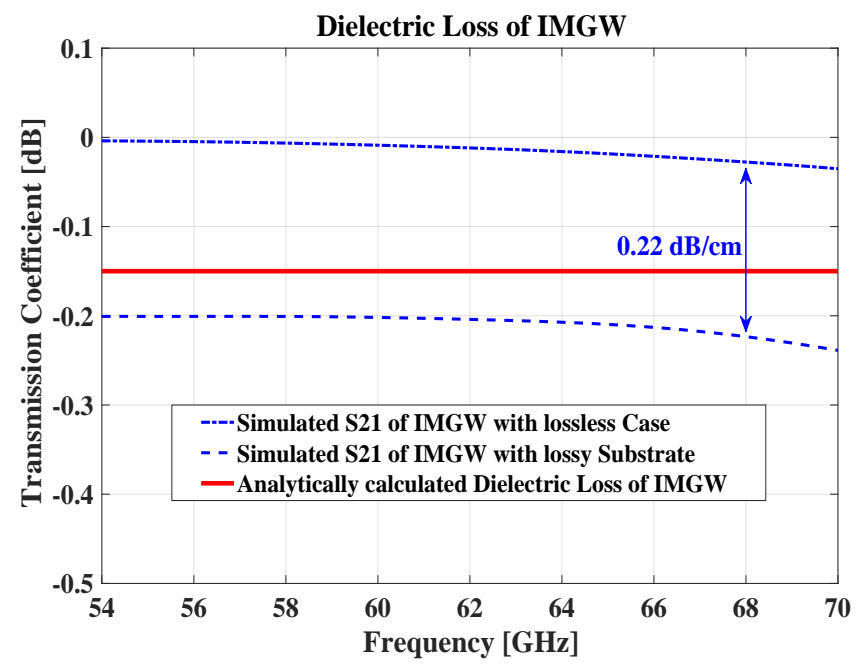

Fig. 16. The Simulated and the calculated dielectric losses of the IMGW. 


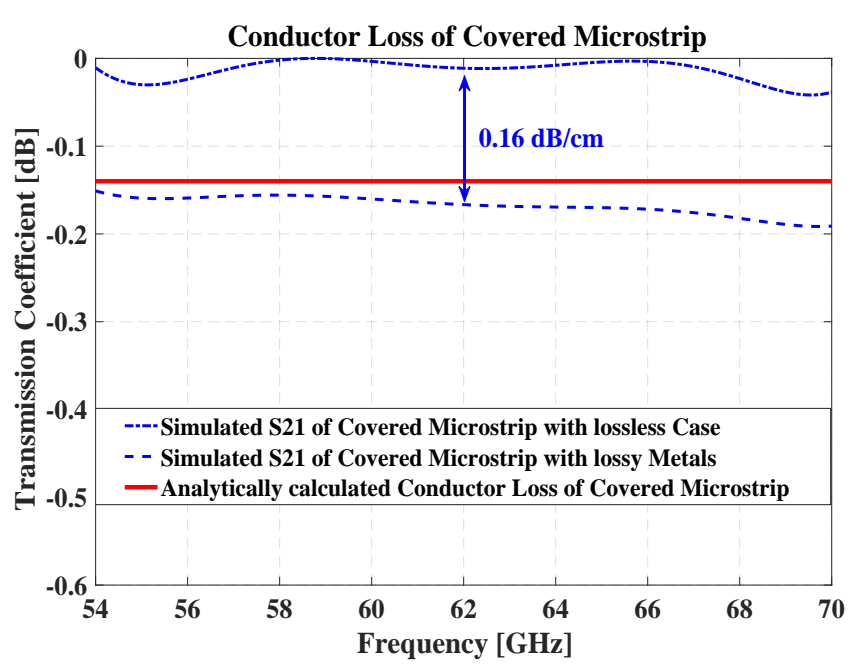

Fig. 17. The Simulated and the calculated conductor losses of the covered microstrip line.

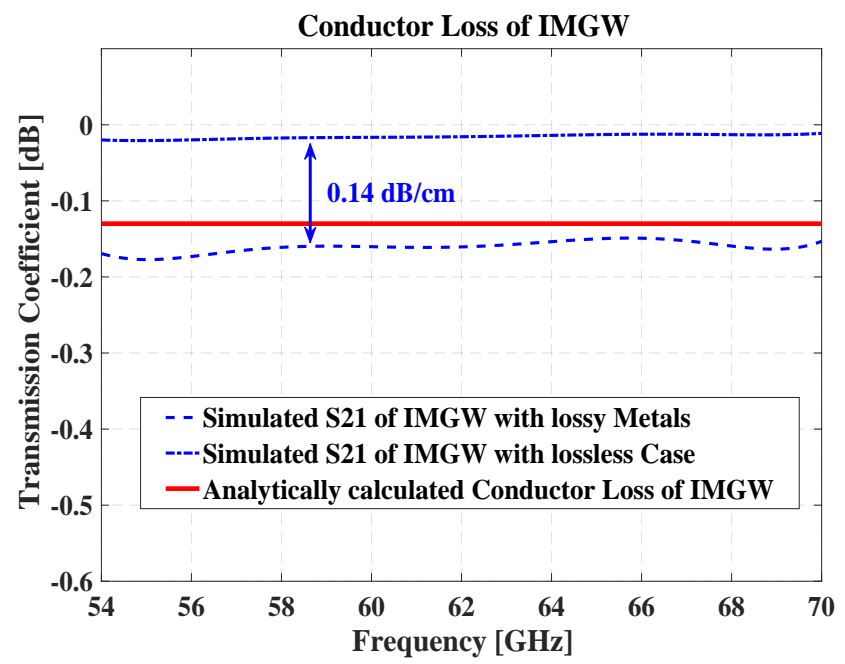

Fig. 18. The Simulated and the calculated conductor losses of the IMGW.

supplies an approximate PMC surface. On the other hand, the similar strategy is utilized to the both structures with a copper microstrip, aluminum plates and lossless substrate so that the difference of the both cases, namely the conductor loss, will be obtained. Fig. 17 and Fig. 18 show the comparisons of the conductor losses. In Fig. 17, the simulated conductor loss of the covered microstrip is about $0.16 \mathrm{~dB} / \mathrm{cm}$, and its calculated value is about $0.13 \mathrm{~dB} / \mathrm{cm}$. In addition, the simulated conductor loss of the IMGW is about $0.14 \mathrm{~dB} / \mathrm{cm}$, and its calculated value is about $0.12 \mathrm{~dB} / \mathrm{cm}$.

For conventional strip line, microtrip and covered microstrip line it is convenient to integrate active components in wireless system. However, they all suffer from high dielectric loss and thereby become a critical problem in mmWs. As shown in Fig. 15, the dielectric loss is always one of key factors for using traditional covered microstrip in mmWs. According to the theory in section II, the unavoidable dielectric loss is characterized by three factors, the electromagnetic field distributions in both air gap and the substrate, the loss tangent value and the thickness of the substrate. Thereby, dielectric loss may be decreased by using thinner substrate materials. Moreover, utilization of substrate materials with low loss tangent value is also an opportunity to decrease the dielectric loss, but the corresponding cost might be increased. Lastly, changing the distribution of the electromagnetic field is a better choice to reduce the dielectric loss and the IMGW is introduced based on the idea. Applying high impedance surface at the bottom of substrate is able to make the electromagnetic wave to propagate in the air gap so that the dielectric loss is significantly decreased, as shown in Fig. 16. Thereby, due to its low loss effect the IMGW is a suitable candidate to integrate Monolithic Microwave Integrated Circuits (MMIC) and design high gain array antenna in mmWs.

\section{CONCLusion}

The variational method has been applied to the IMGW for determining its characteristic impedance, conductor loss and dielectric loss. The analytical solutions have good agreements with the simulated values in CST Microwave Studio, stating that the inverted microstrip gap waveguide technology can be a good candidate for both MMIC integration and high-gain high-efficient array antennas in mmWs communication based on its low dielectric loss property.

\section{REFERENCES}

[1] T. S. Rappaport, J. N. Murdck, and F. Gutierrez, "State of the art in 60$\mathrm{GHz}$ integrated circuits and systems for wireless communications," Proc. IEEE, vol. 99, no. 8, pp. 1390-1436, Aug. 2011.

[2] D. Deslandes and K. Wu, "Integrated microstrip and rectangular waveguide in planar form," IEEE Microwave Wireless Compon. Lett., vol. 11, no. 2, pp. 68-70, Feb. 2001.

[3] B. Maurizio, P. Marco, P. Luca and K. Wu, "On the losses in substrateintegrated waveguides and cavities," International Journal of Microwave and Wireless Technologies, 1(5), 395-401, 2009.

[4] P.-S. Kildal, E. Alfonso, A. Valero-Nogueira, and E. Rajo-Iglesias, "Local metamaterial-based waveguides in gaps between parallel metal plates," IEEE Antennas Wireless Propag. Lett., vol. 8, pp. 84-87, Dec. 2009.

[5] A. Valero-Nogueira, E. Alfonso, J. I. Herranz and P.-S. Kildal, "Experimental demonstration of local quasi-TEM gap modes in singlehard-wall waveguides," IEEE Antennas Wireless Propag. Lett., vol. 19, no. 9, pp. 536-538, Sep. 2009.

[6] P.-S. Kildal, "Three metamaterial-based gap waveguides between parallel metal plates for $\mathrm{mm} / \mathrm{submm}$ waves," in 3rd European Conference on Antennas and Propagation (EUCAP), Berlin, Germany, Mar. 2009, pp. 28-32.

[7] H. Raza, J. Yang and P.-S. Kildal, "Resemblance between gap waveguides and hollow waveguides," IET Microwaves, Antennas and Propagation, vol. 15, no. 7, pp. 1221-1227, Dec. 2013.

[8] P.-S. Kildal, "Artificially soft and hard surfaces in electromagnetics," IEEE Trans. Antennas Propag., vol. 28, no. 10, pp. 1537-1544, Oct. 1990.

[9] H. Raza, J. Yang, P.-S. Kildal, and E. Alfonso, "Microstrip-ridge gap waveguide - study of losses, bends, and transition to WR-15," IEEE Trans. Microw. Theory Tech., vol. 62, no. 9, pp. 1943-1952, Sep. 2014. 
[10] A. Vosoogh, A. A. Brazalez and P.-S.Kildal, "A V-Band inverted microstrip gap waveguide end-coupled bandpass filter," IEEE Microw. Compon. Lett., vol. 26, no. 4, pp. 261-263, Apr. 2016.

[11] A. U. Zaman, P.-S. Kildal and A. A. Kishk, "Narrow-band microwave filter using high-Q groove gap waveguide resonators with manufacturing flexibility and no sidewalls," IEEE Trans. Compon. Packag. Manuf. Technol., vol. 2, no. 11, pp. 1882-1889, Nov. 2012.

[12] M. Rezaee, A. U. Zaman and P.-S. Kildal, "V-band groove gap waveguide diplexer," 9th European Conference on Antennas and Propagation (EuCAP), Lisbon, Portugal, Apr. 2015, pp. 1-4.

[13] A. U. Zaman, M. Alexanderson, T. Vukusic and P.-S. Kildal, "Gap waveguide PMC packaging for improved isolation of circuit components in high-frequency microwave modules," IEEE Trans. Compon. Packag. Manuf. Technol., vol. 4, no. 1, pp. 16-25, Jan. 2014.

[14] D. Zarifi, A. Farahbaksh, A. Uz Zaman and P.-S. Kildal, "Design and fabrication of a wideband high-gain $60-\mathrm{GHz}$ corrugated slot antenna array with ridge gap waveguide distribution layer," IEEE Trans. Antennas Propag., vol. 64, no. 7, pp. 2905-2913, Jul. 2016.

[15] A. U. Zaman and P.-S. Kildal, "Wide-band slot antenna arrays with single-layer corporate-feed network in ridge gap waveguide technology," IEEE Trans. Antennas Propag., vol. 62, no. 6, pp. 2992-3001, Jun. 2014.

[16] A. Vosoogh and P.-S. Kildal, "Corporate-Fed planar 60-GHz slot array made of three unconnected metal layers using AMC pin surface for the gap waveguide," IEEE Microw. Compon. Lett., vol. 15, pp. 1935-1938, Apr. 2016.

[17] J. Liu, A. Vosoogh, A. U. Zaman and J. Yang, "Design and fabrication of a high gain $60-\mathrm{GHz}$ cavity-backed slot antenna array fed by inverted microstrip gap waveguide," IEEE Trans. Antennas Propag., vol. 65, no. 4, pp. 2117-2122, Apr. 2017.

[18] O. Heaviside, Electromagnetic Theory, vol. 1, 1893. Reprinted by Dover, New York, 1950.

[19] S. B. Cohn, "Shielded coupled strip transmission line," IRE Trans. Microwave Theory Tech., vol. 3, pp. 29-38, Oct. 1955.

[20] H. A. Wheeler, "Transmission-line properties of parallel strips separated by a dielectric sheet," IEEE Trans. Microwave Theory Tech., vol. 13, pp. 172-185, Mar. 1965.

[21] D. Park, "Planar transmission lines," IRE Trans. Microwave Theory Tech., vol. 3, part I, pp. 8-12, Apr. 1955; part II, pp. 7-11, Oct. 1955.

[22] K. Foster, "The characteristic impedance and phase velocity of high-q triplate line," Journal of the British Institution of Radio Engineers, vol. 18, no. 12, Dec. 1958.

[23] R. E. Collin, "Characteristic impedance of a slotted coaxial line," IRE Trans. Microwave Theory Tech., vol. 4, pp. 4-8, Jan. 1956.

[24] E. Yamashita and R. Mittra, "Variational method for the analysis of microstrip lines," IEEE Trans. Microwave Theory Tech., vol. 16, pp. 251256, Apr. 1968.

[25] E. Yamashita and K. Atsuki, "Strip line with rectangular outer conductor and three dielectric layers," IEEE Trans. Microwave Theory Tech., vol. 18, no. 5, May, 1970.

[26] T. Itoh and R. Mittra, "Spectral domain approach for calculating the dispersion characteristics of microstrip lines," IEEE Trans. Microwave Theory Tech., vol. 21, pp. 496-499, Jul. 1973.

[27] T. Itoh, "Spectral domain immitance approach for dispersion characteristics of generalized printed transmission lines," IEEE Trans. Microwave Theory Tech., vol. 28, pp. 733-736, Jul. 1980.

[28] R. H. Jansen, "The spectral-domain approach for microwave integrated circuits," IEEE Trans. Microwave Theory Tech., vol. 33, pp. 1043-1056, Oct. 1985.

[29] E. F. Kuester and D. C. Chang, "Theory of dispersion in microstrip of arbitrary width," IEEE Trans. Microwave Theory Tech., vol. 28, pp. 259-265, Mar. 1980.
[30] B. E. Kretch and R. E. Collin, "Microstrip dispersion including anisotropic substrates," IEEE Trans. Microwave Theory Tech., vol. 35, pp. 710-718, Aug. 1987.

[31] R. E. Collin, Field Theory of Guided Waves, Wiley-IEEE Press, New York, Dec. 1990.

[32] C. A. Balanis, Advanced Engineering Electromagnetics, John Wiley \& Sons., 1989.

[33] J. Liu, A. Uz Zaman and P.-S. Kildal, Optimizing numerical port for inverted microstrip gap waveguide in full-wave simulators, 10th European Conference on Antennas and Propagation (EUCAP), Davos, Switzerland, Apr. 2016, pp. 1-5.

[34] J. Wu, Y. J. Cheng, and Y. Fan, "A wideband high-gain highefficiency hybrid integrated plate array antenna for V-Band inter-satellite links," IEEE Trans. Antennas Propag., vol. 63, no. 4, pp. 1225-1233, Apr. 2015.

[35] Based on Communication with Rogers Data Sheet RO4003 Series High Frequency Circuit Materials at $60 \mathrm{GHz}$.

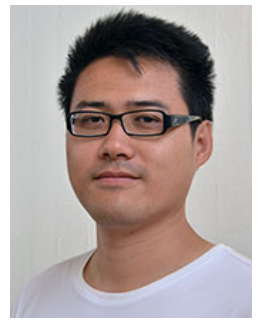

Jinlin Liu (M'17) received the B.Sc. degree in Communications Engineering from the University of Electronic Science and Technology of China (UESTC), Chengdu, China, in 2005, a diploma from Munich University of Technology, Munich, Germany, in 2012 and the Swedish licentiate degree from Chalmers University of Technology, Gothenburg, Sweden, in 2016, respectively. From 2005 to 2007, he was with Intel, Chengdu branch, as a test engineer. From 2012 to 2014, he was with Eurodesign $\mathrm{GmbH}$, Munich, as a PCB test engineer. $\mathrm{He}$ is currently a research assistant with the Communication and Antenna Systems Division, Chalmers University of Technology. His current research interests include fundamental electromagnetic theroy, millimeter-wave planar antennas in general, gap waveguide technology and frequency-selective surfaces. He received the Best Student Paper Award first place at the 2017 International Symposium on Antennas and Propagation, Phuket, Thailand.

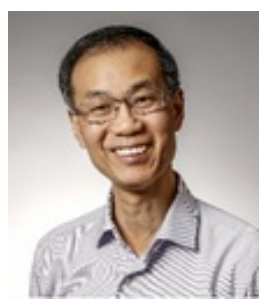

Jian Yang (M'01-SM'10) received the B.Sc. degree in electrical engineering from the Nanjing University of Science and Technology, Nanjing, China, in 1982, the M.Sc. degree in electrical engineering from the Nanjing Research Center of Electronic Engineering, Nanjing, in 1985, and the Swedish Licentiate and Ph.D. degrees from Chalmers University of Technology, Gothenburg, Sweden, in 1998 and 2001, respectively. From 1985 to 1996, he was with the Nanjing Research Institute of Electronics Technology, Nanjing, China, as a Senior Engineer. From 1999 to 2005, he was with the Department of Electromagnetics, Chalmers University of Technology, Gothenburg, Sweden, as a Research Engineer. During 2005 and 2006, he was with COMHAT AB as a Senior Engineer. From 2006 to 2010, he was an Assistant Professor, from 2010 to 2016, he was an Associate Professor with the Department of Signals and Systems, and since 2016 he has been a professor with the Department of Electrical Engineering, Chalmers University of Technology. He has authored and coauthored about 60 journal articles and about 150 conference papers, and more than 10 granted patents. His research interests include $60-140 \mathrm{GHz}$ antennas, terahertz antennas, MIMO antennas, ultra-wideband (UWB) antennas and UWB feeds for reflector antennas, UWB radar systems, UWB antennas in near-field sensing applications, hat-fed antennas, reflector antennas, radome design, and computational electromagnetics. 


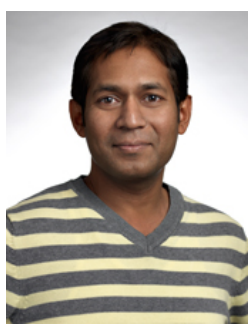

Ashraf Uz Zaman (M'14) was born in Chittagong, Bangladesh. He received the B.Sc. degree in electrical and electronics engineering from the Chittagong University of Engineering and Technology, Chittagong, and the M.Sc. and Ph.D. degrees from the Chalmers University of Technology, Gothenburg, Sweden, in 2007 and 2013, respectively.

$\mathrm{He}$ is currently an Assistant Professor with the Communication and Antenna Systems Division, Chalmers University of Technology. His current research interests include millimeter-wave planar antennas in general, gap waveguide technology, frequency-selective surfaces, microwave passive components, and packaging techniques and integration of MMICs with the antennas. 